\title{
EFFECT OF TEMPERATURE AND COMPRESSION/DECOMPRESSION RATES ON HIGH PRESSURE INACTIVATION OF LISTERIA
}

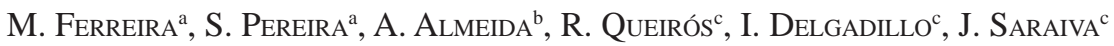 \\ and A. CUNHA ${ }^{\text {b* }}$ \\ ${ }^{a}$ Biology Department, University of Aveiro, Campus de Santiago, 3810-193 Aveiro. Portugal \\ biology Department and CESAM, University of Aveiro, Campus de Santiago, 3810-193 Aveiro. Portugal \\ ${ }^{\mathrm{c}}$ Chemistry Department and QOPNA, University of Aveiro, Campus de Santiago, 3810-193 Aveiro. Portugal
}

(Received: 11 December 2014; accepted: 9 March 2015)

The main objective of the present study was to evaluate the effect of temperature and different compression/ decompression rates on the efficiency of Listeria inactivation by HPP.

Stationary phase cultures of Listeria innocua were subjected to $300 \mathrm{MPa}$ for 5 min at 4 , 10 , and $20^{\circ} \mathrm{C}$ using different compression and decompression rates. Inactivation was more efficient at low temperature and with lowest compression and decompression rates (1.5 $\mathrm{MPa} \mathrm{s}^{-1}$ and $3.2 \mathrm{MPa} \mathrm{s}^{-1}$, respectively).

Kinetics of pressure building up and decompression, as well as temperature, have a significant impact on the outcome of Listeria inactivation by HPP. The results may contribute to the design of HPP protocols that ensure food safety, while preserving nutritional and organoleptic properties better.

Keywords: high-pressure processing (HPP), food safety, Listeria, compression and decompression rates, temperature

High-pressure processing (HPP) has been considered a promising alternative to conventional thermal pasteurization of food products, because microbial death is achieved without using heat, chemical additives, or preservatives, and natural flavour and texture of the food are maintained (RAstogi et al., 2007; Sousa et al., 2013). Temperature and pressurization parameters, such as compression and decompression rates, may significantly influence the process outcome. Adiabatic heating occurs as pressure builds up and therefore, when very high pressures are applied, the achieved end-temperature may enhance microbial inactivation (RAMirez et al., 2009).

Vegetative bacterial cells have been reported as less susceptible to HPP at temperatures between 20 and $30{ }^{\circ} \mathrm{C}$ (RAso \& Barbosa-CÁnovas, 2003) than at temperatures above or below this range. The influence of compression and decompression rates on different microorganisms and in different food matrices is still controversial. Enhanced inactivation of vegetative bacterial cells (NomA et al., 2002; CHAPLEAu et al., 2006) and bacterial endospores (HAYAKAWA et al., 1998) with fast compression and/or decompression has been demonstrated but the inverse effect has also been reported (CHAPLEAU et al., 2006). Some experiments with Listeria innocua failed to demonstrate any significant effect of the pressurization ramp in the final outcome of inactivation (RADEMACHER et al., 2002). It is most likely that the effect of compression and decompression kinetics is strongly modulated by other physical and chemical factors, namely maximum pressure and adiabatic heating. The main objective of

\footnotetext{
* To whom correspondence should be addressed.

Phone: +351-234-370784; fax: +351-234-426408; e-mail: acunha@ua.pt
} 
this work was to assess the importance of temperature and compression/decompression rates as determinant parameters on the efficiency of $L$. innocua inactivation by HPP, in order to provide scientific basis for the design of more efficient inactivation protocols.

\section{Materials and methods}

Listeria innocua (NCTC 10528) was used as an amenable surrogate for the pathogenic $L$. monocytogenes. Fresh stationary phase cultures were prepared in Tryptic Soy Broth (TSB, Liofilchem) before each inactivation assay and distributed in sterile compression microtubes (Microtube PE $0.5 \mathrm{ml}$ Beckmann). In order to set out the most adequate treatment conditions for subsequent experiments, in which a significant but incomplete inactivation was intended, relation between microorganism survival and pressure was initially established. Samples were subjected to $100,200,300,400,500$, and $600 \mathrm{MPa}$, for $5 \mathrm{~min}$, at room temperature $\left(22-24{ }^{\circ} \mathrm{C}\right)$ in a Unipress Equipment (Model U33). Pressure holding time did not include compression and decompression time. After compression, samples were kept in melting ice until further processing.

To assess temperature influence, a set of HPP assays was conducted at 4,10 , and $20^{\circ} \mathrm{C}$ with a pressure value of $300 \mathrm{MPa}$ and a 5-min holding time. Compression/decompression rates were set to intermediate level.

In order to evaluate the influence of compression and decompression rates, bacterial suspensions were submitted to slow, intermediate, and fast compression and decompression. Two different pressure values were tested, $300 \mathrm{MPa}$ and $400 \mathrm{MPa}$. Pressure was applied for 1 or $5 \mathrm{~min}$, at $20^{\circ} \mathrm{C}$. The increase and decrease in pressure were linear with time. Compression and decompression rates, expressed as MPa s${ }^{-1}$, were calculated by dividing holding pressure value by time (s) required to reach it (compression) or to its complete release (decompression).

For determination of the concentration of viable cells in the untreated control $\left(\mathrm{N}_{0}\right)$ and after the different treatments $\left(\mathrm{N}_{\mathrm{i}}\right)$, aliquots were serially diluted in Ringer solution (Merck Millipore) and pour-plated in triplicate in Tryptic Soy Agar (TSA, Liofilchem). Plates were incubated for $48 \mathrm{~h}$ at $37{ }^{\circ} \mathrm{C}$ and colonies were counted in replicates of the most suitable dilution. The concentration of viable cells $\left(\mathrm{CFU} \mathrm{ml}{ }^{-1}\right)$ was determined from the average of replicates of the most convenient dilution, corrected for the dilution factor. Number of decimal reduction (NDR) was calculated as the logarithmic reduction of the concentration of colony forming units, using the expression NDR $=\log _{10}\left(\mathrm{~N}_{0} / \mathrm{N}_{\mathrm{i}}\right)$.

All experimental conditions were tested in 3 or 4 independent assays including 3 analytical replicates. NDR for each condition was calculated as the average of NDR values of each set of independent assays. Significant differences between treatments were assessed by ANOVA, after verification of normal distribution and homogeneity of variances. When normality was not observed, the significance of the differences was evaluated by KruskalWallis test. Whenever significant differences were detected, post-hoc bilateral Tukey's test was applied. All statistical analyses were conducted with SPSS Statistics 20 package.

\section{Results and discussion}

The results of preliminary experiments (Fig. 1) were used to determine the conditions required for significant but incomplete inactivation, to be used in subsequent tests. Holding 
time was chosen from preliminary assays (data not shown) for causing significant inactivation with relatively low pressure (300 and $400 \mathrm{MPa}$ ). Between 100 and $400 \mathrm{MPa}$, there was an increase in inactivation efficiency with increasing pressure (ANOVA, $\mathrm{P}<0.05$ ). NDR varied between $0.3 \pm 0.29$ with $100 \mathrm{MPa}$, and $9.2 \pm 0.00$ with $400 \mathrm{MPa}$. At $300 \mathrm{MPa}$ a significant, although incomplete inactivation $(4.4 \pm 0.07 \mathrm{log})$ was obtained, this pressure value was selected for further assays in order to detect variations in inactivation efficiency. The results obtained are coherent with the information available in the literature. A direct relation between pressure and inactivation has been demonstrated for Listeria, as well as for other microorganisms (Dogan \& ERKMEN, 2004; Rendueles et al., 2011). Overall, NDR are in agreement with values reported for L. innocua in other HPP studies (BuzRUL et al., 2008; GudBJORnSDOtTir et al., 2010; Evrendilek \& Balasubramaniam, 2011), even though those values vary considerably with different compression conditions and matrices into which the microorganism is inoculated.

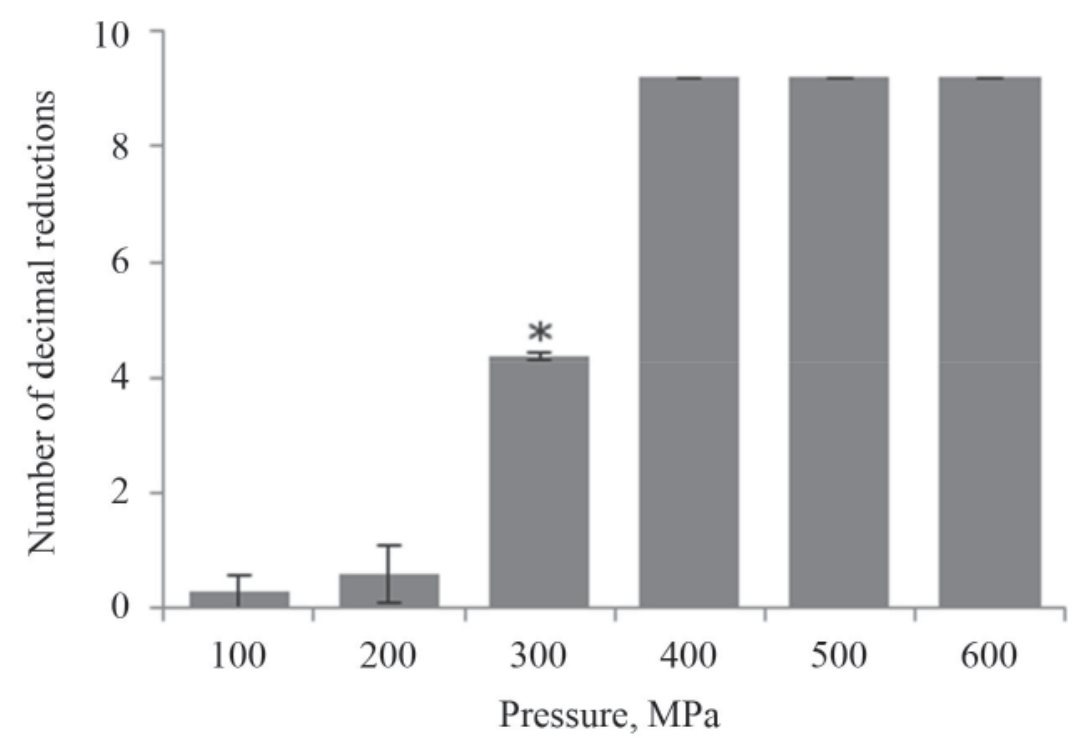

Fig. 1. Number of decimal reductions (NDR) calculated for preliminary tests of Listeria innocua inactivation in assays conducted at $22-24^{\circ} \mathrm{C}$ with a 5-min holding time and intermediate compression and decompression rates, 6-7 and 13-15 $\mathrm{MPa} \mathrm{s}^{-1}$, respectively. Columns represent the average NDR calculated from 3 independent assays and the error bars represent the standard deviation. * indicates statistically significant differences $(\mathrm{P}<0.05)$.

The evaluation of the effect of temperature during HPP treatment on the inactivation efficiency shows that, at $300 \mathrm{MPa}$, susceptibility to pressure decreased with increasing temperature (Fig. 2). Susceptibility at $4{ }^{\circ} \mathrm{C}(\mathrm{NDR}=5.6 \pm 0.25)$ and at $10{ }^{\circ} \mathrm{C}(\mathrm{NDR}=5.1 \pm 0.32)$ was not significantly different (ANOVA, $\mathrm{P}>0.05$ ). A significantly lower inactivation (ANOVA, $\mathrm{P}<0.05)$ was obtained at $20^{\circ} \mathrm{C}(3.0 \pm 0.46)$.

The effect of temperature on the efficiency of HP inactivation has been studied for a wide range of microorganisms, but more often for temperature ranges above $25{ }^{\circ} \mathrm{C}$. As a general trend, inactivation efficiency increases when temperature rises above the optimal growth temperature (Alpas et al., 2000; PATtERson, 2005). HPP studies below room 
temperature are still scarce and this information becomes particularly critical when the inactivation of microorganisms able to grow under refrigeration temperatures, such as Listeria monocytogenes (BAJARD et al., 1996), is intended.

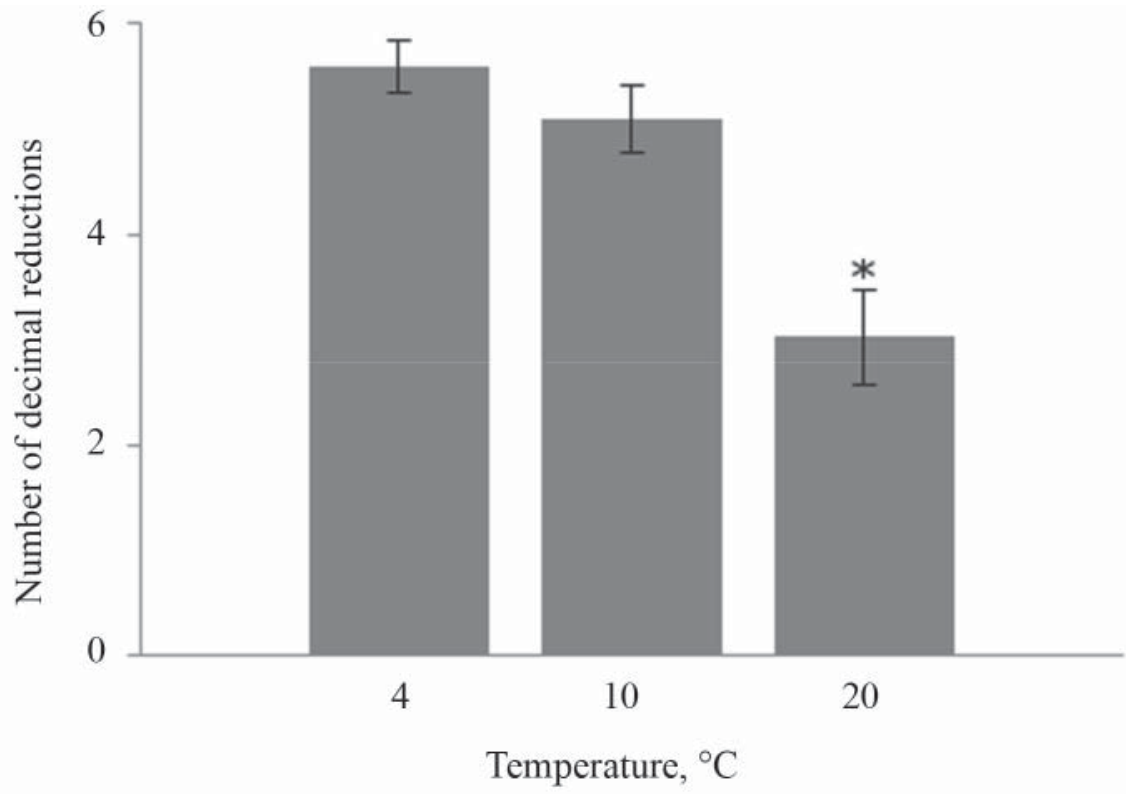

Fig. 2. Inactivation of Listeria innocua by HPP at different temperatures, with pressurization at $300 \mathrm{MPa}$, 5-min holding time, and intermediate compression and decompression rates (6.4 and $12.9 \mathrm{MPa} \mathrm{s}^{-1}$, respectively). Results correspond to the average of 3 independent assays and the error bars represent the standard deviation. * indicates statistically significant differences $(\mathrm{P}<0.05)$.

The results of the present work indicate that the inactivation of L. innocua in TSB was highest when pressurization was conducted below room temperature $\left(4-10^{\circ} \mathrm{C}\right)$. Similar results have been obtained for Listeria species inoculated in meat (CARLEz et al., 1993), milk (Gervilla et al., 2000), and vegetables (Arroyo et al., 1999). In some cases, the NDR obtained at $4{ }^{\circ} \mathrm{C}$ can only be exceeded when pressurization temperature is higher than $50{ }^{\circ} \mathrm{C}$ (CARLEz et al., 1993).

The kinetics of pressure increase and decrease is an important operational parameter in HP processing of foods, due to its implications on adiabatic heating and cooling and on the eliciting of adaptive responses on microorganisms. Three different compression/ decompression rates - low, intermediate, and high - within the operational limits of the equipment were tested. The exact values calculated for each experimental condition are presented in Table 1. 
Table 1. Compression and decompression rates calculated for independent HPP experiments conducted with Listeria innocua.

\begin{tabular}{lcc}
\hline \multirow{2}{*}{ Pressure values, MPa } & \multicolumn{2}{c}{ Kinetic parameters, MPa s $^{-1}$} \\
\cline { 2 - 3 } 300 & Compression rate & Decompression rate \\
\hline \multirow{2}{*}{400} & 1.5 & 3.2 \\
& 6.4 & 12.9 \\
& 10.7 & 21.0 \\
& 1.6 & 3.8 \\
& 6.9 & 14.8 \\
& 11.9 & 23.8 \\
\hline
\end{tabular}

In the assays conducted at $300 \mathrm{MPa}$, NDR calculated for different treatments were not significantly different with 1 -min holding time (ANOVA, $\mathrm{P}>0.05$ ), but were significantly higher with slow compression and decompression ( $\mathrm{NDR}=3.3 \pm 0.48$ ), in relation to medium and fast compression/decompression rates, when holding time was extended to $5 \mathrm{~min}$ (ANOVA, $\mathrm{P}<0.05$ ) (Fig. 3). It has been suggested that slow compression and decompression leads to higher inactivation than any other combination of fast and slow compression and decompression, although experiments were conducted with $B$. subtilis spores, at different temperatures, and in different matrices (SYED et al, 2012). These results are apparently counter intuitive, considering that a prolonged compression period would elicit stress responses and reduce susceptibility (SMELT, 1998). In the present study, the overall effect combines the damage caused during compression and decompression, and the general trend

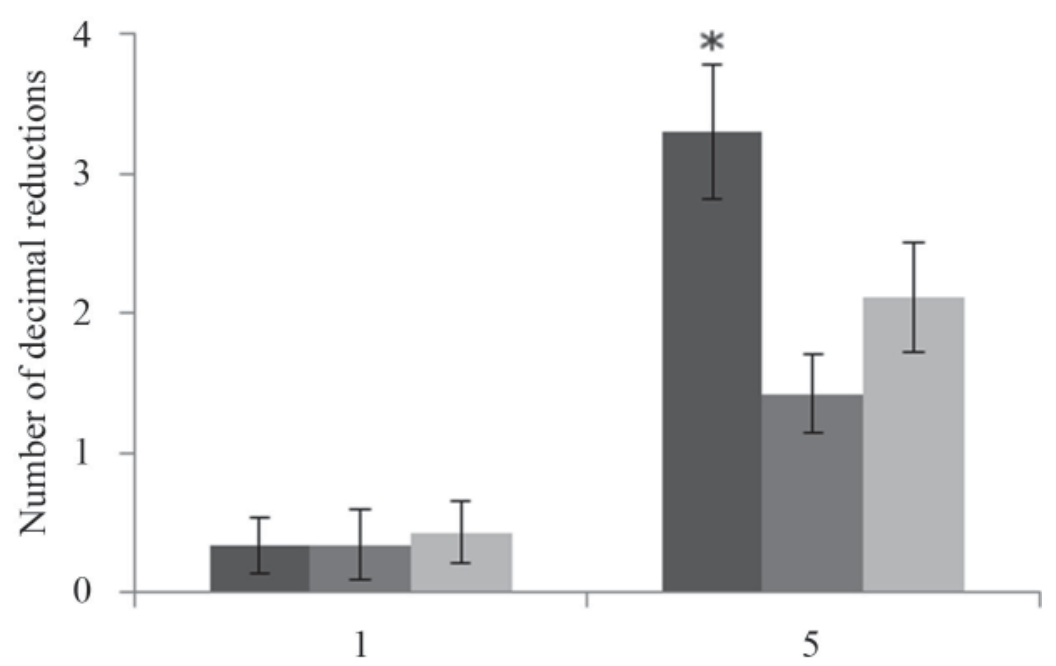

Holding time, $\min$

Fig. 3. Number of decimal reductions (NDR) obtained in assays of Listeria innocua inactivation at 300 MPa with different compression and decompression rates and holding times of 1 or $5 \mathrm{~min}$. Columns represent the average results obtained in 4 independent assays, and error bars represent the standard deviation.* indicates statistically significant differences $(\mathrm{P}<0.05)$. $\square$ : low rate; $\square$ : intermediate rate; $\square$ : high rate 
of higher NDR values with slowest compression and decompression may be interpreted as an indication that $L$. innocua inactivation, such as proposed for other microorganisms, is more affected by decompression rate than by compression rate (NomA et al., 2002). Results on the effect of pressurization kinetics are still controversial, and difficulties in establishing comparisons arise from very different experimental conditions, namely in terms of pressure and temperature, because most studies are conducted in maximum inactivation conditions. When a pressure of $400 \mathrm{MPa}$ was used, the effect of compression/decompression rates could not be detected, because a very high NDR value (8.1 \pm 1.03$)$, corresponding to near complete inactivation, was obtained (Fig. 4).

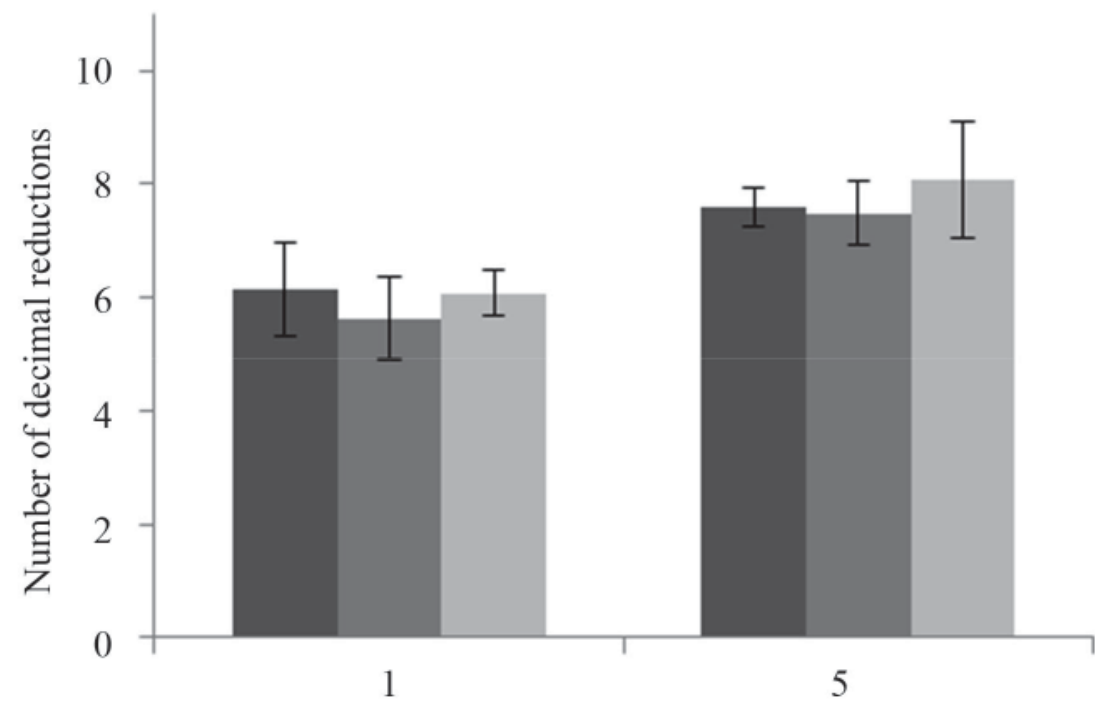

Holding time, min

Fig. 4. Number of decimal reductions (NDR) obtained in assays of Listeria innocua inactivation at $400 \mathrm{MPa}$ with different compression and decompression rates and holding times of 1 or $5 \mathrm{~min}$. Columns represent the average results obtained in 4 independent assays and error bars represent the standard deviation.

$\square$ : low rate; 0 : intermediate rate; 1 : high rate

From the results obtained in preliminary assays, $300 \mathrm{MPa}$ pressure was selected to be used in subsequent tests by causing highest significant incomplete inactivation. In the initial experiments, $5 \mathrm{~min}$ at $400 \mathrm{MPa}$ caused the complete inactivation of the cell suspension, but later, in the experiments with variable compression/decompression rates, the same conditions failed to cause complete inactivation. More strikingly, although the results from each set of experiments are highly reproducible, there were marked differences in NDR values obtained in different sets of experiments performed under similar conditions (pressurization to 300 MPa for 5 min at approximately $20^{\circ} \mathrm{C}$ with intermediate compression/decompression rates). In the first set of experiments conducted to establish a relation between pressure and inactivation efficiency, a NDR of $4.4 \mathrm{log}$ was obtained. When testing the effect of temperature, inactivation was lower (3.0 log), and in later experiments, addressing the effect of compression/ decompression rates, NDR was as low as $1.5 \mathrm{log}$. In this work, cultivation medium and 
conditions were standardized and growth phase was rigorously controlled in order to obtain stationary phase cells displaying the lowest susceptibility to high pressure inactivation. Therefore, the decrease in susceptibility during the time span in which experiments were performed cannot be attributed to variations in experimental conditions. Considering that cold and pressure may trigger similar adaptations in terms of membrane composition and function (RusselL, 2002), it is possible that some changes in susceptibility to pressure may have occurred in stock cultures. Once stock cultures used in this work were kept in cold $\left(4^{\circ} \mathrm{C}\right)$ between assays, some adaptation may have occurred during storage. The development of resistance to HPP in Listeria during cold storage is a pertinent issue that must be addressed in future work.

\section{Conclusions}

The results of present work demonstrate that, in addition to the known dependence on pressure and holding time, efficiency of high-pressure inactivation of Listeria innocua is also affected by processing temperature and compression/decompression kinetics. Application of a low compression rate followed by high-pressure at $300 \mathrm{MPa}$ for $5 \mathrm{~min}$, and a low decompression rate, as well as a low processing temperature $\left(10-20^{\circ} \mathrm{C}\right)$ lead to enhanced $L$. innocua inactivation. These results may provide the basis for HPP protocols that better preserve food nutritional properties, texture, and flavour, although the eventual development of resistance to HPP during cold storage must be carefully considered.

Thanks are due to FCT-Portugal, QREN, FEDER, and COMPETE for funding the research units QOPNA (PEst-C/ QUI/UI0062/2013) and CESAM (PEst-C/MAR/LA0017/2011). The authors are grateful to Prof. Paula Teixeira from Escola Superior de Biotecnologia da Universidade Católica Portuguesa, Porto for providing the Listeria innocua strain used in this study.

\section{References}

Alpas, H., Kalchayanand, N., Bozoglu, F. \& Ray, B. (2000): Interactions of high hydrostatic pressure, pressurization temperature and $\mathrm{pH}$ on death and injury of pressure-resistant and pressure-sensitive strains of foodborne pathogens. Int. J. Food Microbiol., 60, 33-42.

Arroyo, G., Sanz, P. \& Prestamo, G. (1999): Response to high-pressure, low-temperature treatment in vegetables: determination of survival rates of microbial populations using flow cytometry and detection of peroxidase activity using confocal microscopy. J. Appl. Microbiol., 86, 544-556.

Bajard, S., Rosso, L., Fardel, G. \& Flandrois, J. (1996): The particular behaviour of Listeria monocytogenes under sub-optimal conditions. Int. J. Food Microbiol., 29, 201-211.

Buzrul, S., Alpas, H., Largeteau, A. \& Demazeau, G. (2008): Inactivation of Escherichia coli and Listeria innocua in kiwifruit and pineapple juices by high hydrostatic pressure. Int. J. Food Microbiol., 124, 275-278.

Carlez, A., Rosec, J-P., Richard, N. \& Cheftel, J-C. (1993): High pressure inactivation of Citrobacter freundii, Pseudomonas fluorescens and Listeria innocua in inoculated minced beef muscle. LWT - Food Sci. Technol., 26, 357-363.

Chapleau, N., Ritz, M., Delépine, S., Jugiau, F., Federighi, M. \& de Lamballerie, M. (2006): Influence of kinetic parameters of high pressure processing on bacterial inactivation in a buffer system. Int. J. Food Microbiol., 106, 324-330.

Dogan, C. \& ERKmen, O. (2004): High pressure inactivation kinetics of Listeria monocytogenes inactivation in broth, milk, and peach and orange juices. J. Food Eng., 62, 47-52. 
Evrendilek, G.A. \& Balasubramaniam, V. (2011): Inactivation of Listeria monocytogenes and Listeria innocua in yogurt drink applying combination of high pressure processing and mint essential oils. Food Control, 22, 1435-1441.

Gervilla, R., Ferragut, V. \& Guamis, B. (2000): High pressure inactivation of microorganisms inoculated into ovine milk of different fat contents. J. Dairy Sci., 83, 674-682.

Gudbjornsdottir, B., Jonsson, A., Hafsteinsson, H. \& Heinz, V. (2010): Effect of high-pressure processing on Listeria spp. and on the textural and microstructural properties of cold smoked salmon. LWT - Food Sci. Technol., 43, 366-374.

Hayakawa, I., Furukawa, S., Midzunaga, A., Horiuchi, H., Nakashima, T., Fujio, Y., Yano, Y., Ishikura, T. \& Sasaki, K. (1998): Mechanism of inactivation of heat-tolerant spores of Bacillus stearothermophilus IFO 12550 by rapid decompression. J. Food Sci., 63, 371-374.

Noma, S., SHIMODA, M. \& HaYAKAWA, I. (2002): Inactivation of vegetative bacteria by rapid decompression treatment. J. Food Sci., 67, 3408-3411.

Patterson, M. (2005): Microbiology of pressure-treated foods. J. Appl. Microbiol., 98, 1400-1409.

Rademacher, B., Werner, F. \& Pehl, M. (2002): Effect of the pressurizing ramp on the inactivation of Listeria innocua considering thermo fluid dynamical processes. Innov. Food Sci. Emerg., 3, 19-24.

Ramirez, R., Saraiva, J., Lamela, C.P. \& Torres, J.A. (2009): Reaction kinetics analysis of chemical changes in pressure-assisted thermal processing. Food Eng. Rev., 1, 16-30.

RAso, J. \& BARBosa-CÁNovas, G.V. (2003): Nonthermal preservation of foods using combined processing techniques. CRC Cr. Rev. Food Sci., 43, 265-285.

Rastogi, N., Raghavarao, K., Balasubramaniam, V., Niranjan, K. \& Knorr, D. (2007): Opportunities and challenges in high pressure processing of foods. CRC Cr. Rev. Food Sci., 47, 69-112.

Rendueles, E., Omer, M., Alvseike, O., Alonso-Calleja, C., Capita, R. \& Prieto, M. (2011): Microbiological food safety assessment of high hydrostatic pressure processing: A review. LWT - Food Sci. Technol., 44, 12511260.

Russell, N.J. (2002): Bacterial membranes: the effects of chill storage and food processing. An overview. Int. J. Food Microbiol., 79, 27-34.

Smelt, J. (1998): Recent advances in the microbiology of high pressure processing. Trends Food Sci. Technol., 9, $152-158$.

Sousa, S., Delgadillo, I. \& Saraiva, J. (2013): Human milk composition and preservation: evaluation of highpressure processing as a non-thermal pasteurisation technology. CRC Cr. Rev. Food Sci., DOI: 10.1080/10408398.2012.753402.

Syed, Q-A., Reineke, K., Saldo, J., Buffa, M., Guamis, B. \& Knorr, D. (2012): Effect of compression and decompression rates during high hydrostatic pressure processing on inactivation kinetics of bacterial spores at different temperatures. Food Control, 25, 361-367. 